\title{
Monitoring eye movements during PA learning under three display conditions'
}

\author{
P. D. MCCORMACK, G. D. CLEMENCE, W. \\ H. TYMM, and M. A. MALABRE, Carleton \\ University, Ottawa, Canada
}

Data of earlier investigations, where eye movements were monitored during the learning of verbal paired associates, have been consistent with a two-stage notion. The present study was initiated with the purpose of determining the extent to which these findings might have been contaminated by artifact arising from presentation of the stimulus on the left and of the response on the right of the display. Three conditions were employed, with the stimulus either above, below, or to the right of the response. It was concluded that the use of a standard left-right display leads to phenomena which are consistent with the two-stage notion but which, at the same time, reflect a reading habit artifact.

While learning verbal paired associates (PA) by the study-test method, Ss attend more to the response than to the stimulus during early learning trials. As learning progresses, stimulus-viewing becomes more frequent and eventually predominates (e.g., McCormack \& Haltrecht, 1966). An increase in number of eye movements over trials has also been observed. These phenomena, obtained in settings where the stimulus is on the left and the response on the right of the display, have been interpreted as being consistent with the two-stage notion of PA learning (Underwood \& Schulz, 1960), the first stage being one of response consolidation whereas the second involves stimulus. response hook-up.

The data of one study in which eye movements were monitored (McCormack, Haltrecht, \& Hannah, 1966), however, indicated that the preponderance of response viewing observed in early learning trials might be due, at least in part, to artifact such as a left-to-right reading habit. The present investigation was therefore initiated with the purpose of determining the extent to which earlier findings might have been contaminated by an artifact arising from employment of a left-right horizontal display. Ss were therefore required to learn PA lists under each of three conditions. Two of these involved a vertical display, with the stimulus presented above the response in one case and below it in the other. The third condition was one involving a horizontal display with the stimulus on the right and the response on the left. Should the usual fixation functions be obtained with these modes of presentation, the data would again be consistent with the two-stage notion. On the other hand, if Ss exhibit increased response and decreased stimulus viewing over trials, particularly where the display demands right-to-left scanning, the earlier findings would have to be reassessed in the light of a demonstrated artifact. A third possibility exists, one where Ss might show no reliable stimulus or responsefixation trends over trials. Should data of this nature be obtained, it would have to be concluded that employment of a standard display reflects the transition from Stage 1 to Stage 2 as well as artifact effects attributable to left-to-right scanning habits.

\section{EXPERIMENT 1}

Subjects and Apparatus

Subjects were 48 students of introductory psychology. The PA list consisted of seven pairs of CVCs with $\mathrm{m}^{\prime}$ values (Noble, 1961) within the range 1.80-1.93. The list was displayed by means of a timercontrolled Carousel unit fixed at a distance of $85 \mathrm{~cm}$ behind a translucent screen. $S$ was located $62 \mathrm{~cm}$ in front of the screen with his head immobilized. Eye movements were monitored by means of an Eye Movement Recorder (Itek, Model V-1164), consisting of a Pathe (Professional Reflex-16AT) camera loaded with $100 \mathrm{ft}$ of $16-\mathrm{mm}$ film moving at 16 frames/sec, and an optical system that transmits a spot of light from the cornea to the film. The film, when developed, shows the PA slides and, superimposed on each, a bright circular spot indicating the approximate position of actual fixation. The data were analyzed frame by frame by examining the CVC pairs through a film-viewer.

\section{Procedure}

Fifteen Ss were assigned to Condition $\mathrm{A}$ where the stimulus appeared above the response. These $S s$ were instructed to study the top and bottom items and were told that they would be required to call out the bottom item whenever the top item was presented alone. The display was reversed for Condition B, with the stimulus presented below the response. Seventeen Ss were assigned to this group and were instructed to produce the upper item whenever the lower item appeared alone. In Condition $\mathrm{R}$ the stimulus appeared to the right of the response, and each of $16 \mathrm{Ss}$ was required to call out the left item whenever the CVC on the right was presented alone.

On any given trial, the seven CVC pairs were presented consecutively. During this study phase, $S$ was instructed to examine the pairs in an attempt to learn them. Following a 2-sec interval, the seven stimuli were presented, with $S$ attempting to recall the appropriate responses during this test phase. Slides changed every $2 \mathrm{sec}$, the intertrial interval was $2 \mathrm{sec}$, and a 10-sec delay was introduced at the end of the fourth trial so that the Carousel magazine could be returned to its original position. The list was presented in each of four different random orders in an attempt to minimize serial learning and the session was terminated after the completion of the eighth trial.

Results

The main findings for Condition A, where the stimulus appeared above the response, are summarized in Fig. 1. Trials are shown along the abscissa while the ordinate represents mean number of frames per syllable pair for those slides where the stimulus and the response appeared together. The broken function indicates response frames while the solid one depicts stimulus frames. The findings portrayed by Fig. 1 were evaluated by means of analysis of variance techniques. A statistically dependable Conditions ( $\mathrm{S}$ vs $\mathrm{R}$ ) by Trials interaction was revealed $[F(7,98)=3.18$, $p<.01]$, indicating increased viewing of the stimulus in relation to the response over trials. The eye-movement data were also subjected to analysis of variance, and a reliable trials effect $[F(7,98)=8.60$ $\mathrm{p}<.001$ ] was obtained. This increase in number of eye movements over trials reflects a transition from S-R to S-R-S viewing.

A statistical analysis of the fixation functions of Condition $B$, where the stimulus appeared below the response during study trials, revealed no dependable main effects or interactions. An analysis of the eye-movement data, however, demonstrated a reliable trials effect $[F(7,112)=5.40, p<.001]$, with Ss showing a transition from R-S to R-S-R viewing patterns. For Condition $R$, where the stimulus appeared to the right of the response, no reliable effects were revealed in an overall analysis of either the fixation or of the eye-movement data. Under this condition eye movements were of the $R_{2} S$ variety throughout the eight trials.

EXPERIMENT ?

Under Conditions $\mathrm{B}$ and $\mathrm{R}$ of Experiment 1 , Ss looked first to the response and then to the stimulus during early learning trials, with $S s$ of Condition B later exhibiting an R-S-R type of viewing pattern. These data, unlike those of Condition $A$ or those of the standard horizontal display condition where eye movements are of the 


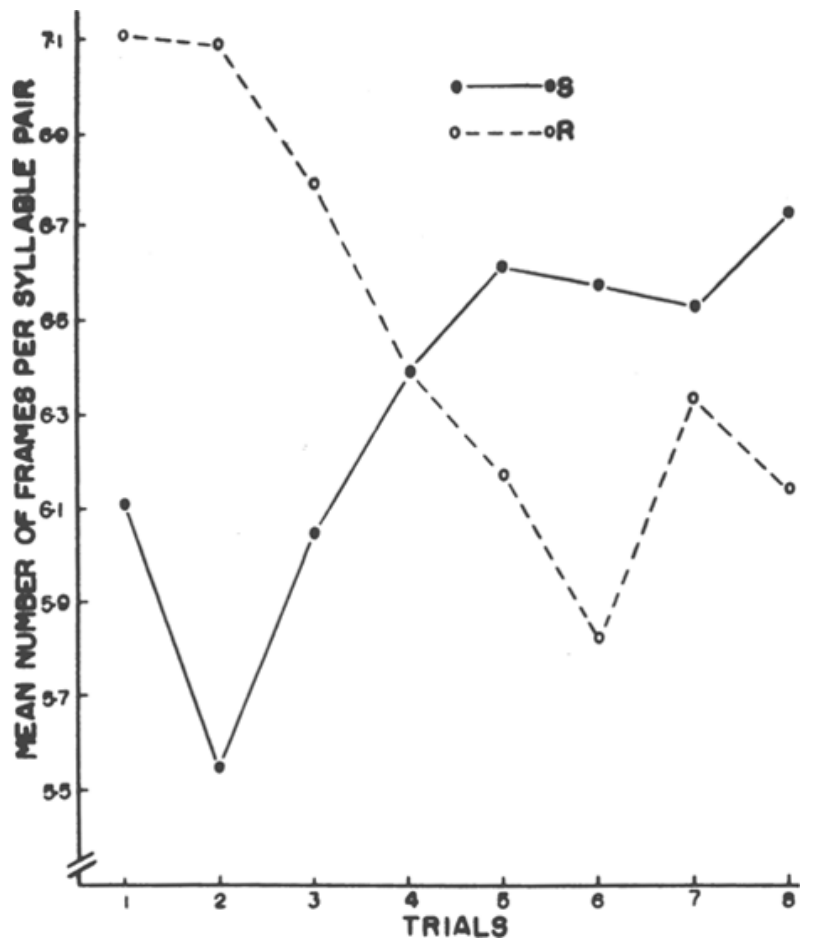

S-R or S-R-S variety, led the investigators to suspect that the instructions were not properly understood, Ss possibly treating the stimulus as a response and thus learning in a backward direction. Experiment 2 was conducted, therefore, with the purpose of making the instructions more explicit for the $B$ and $R$ display conditions.

Subjects were 30 students of introductory psychology, 15 assigned to each of Conditions B and R. It was made clear at the outset which CVC was the stimulus and which was the response, and that $S$ would be required to produce the latter in the presence of the former. Sixteen trials were given, with the first being a nonfilmed practice trial followed by a repetition of instructions for purposes of clarification. In all other respects, the procedure was like that of Experiment 1.

\section{Results}

The fixation and eye-movement data for Condition B were identical to those of Experiment 1. There were no statistically dependable main effects or interactions arising from analysis of the fixation data, whereas the eye-movement analysis revealed a transition from R-S to R-S-R viewing; for trials, $F(14,196)=2.14, p<.025$. As in the first experiment, there were no statistically dependable effects in either the fixation or in the eye-movement data of Condition $\mathbf{R}$. The eye-movement patterns, however, were mostly of the $S-R$ variety as contrasted with a preponderance of R-S viewing in lixperiment 1.

\section{DISCUSSION}

For displays where the stimulus is presented to the left of or above the response, response viewing predominates during early learning trials but is later superseded by stimulus viewing. This transition is accompanied by changes in eye-movement patterns from those of an S-R to those of an S-R-S variety. These data are consistent with the notion of a transition from a stage of response consolidation to one of S-R hook-up. However, when the stimulus is presented below or to the right of the response, no systematic changes over trials are observed in the fixation function data. Furthermore, these displays produce atypical eye-movement patterns. With the stimulus below the response, the patterns show an R-S to an R-S-R transition, and with the stimulus to the right of the response $R-S$ patterns are observed under standard instructions, but these change to those of the $S-R$ type when instructions are made more explicit.

Examination of the appropriate error mean squares for testing the reliability of the Conditions by Trials interaction for the fixation data of each of the five groups of Ss, indicates that the $B$ and $R$ displays produce more variable behavior than either of the other two displays. With the stimulus above the response, the error mean square was 1.63 , its magnitude being comparable to mean squares obtained in investigations where the stimulus was presented to the left of the response. In the $B$ condition, these mean squares were 2.73 and 1.94 for Experiments 1 and 2, respectively. With the stimulus on the right, the corresponding mean square ratios increased to 3.00 and 3.64.
Fig. 1. Mean number of frames per syllable prir as a function of trials for Condition A.

An analysis of variance was also performed on total number of correct responses from Trials $2-8$ for each of the five groups of Ss in Experiments 1 and 2. Although no statistically reliable groups effect was revealed, the means were in a direction consistent with the fixation and eye-movement data. With the stimulus presented above the response, the condition where the findings were like those of the standard left-right display, the mean number of correct responses was 13.4. In Experiment 2 , where the stimulus was presented to the right of the response and where the more explicit instructions led to a reversal from $R-S$ to $S-R$ viewing, the mean number of correct responses was 12.2 . This condition in Experiment 1 resulted in a mean of only 10.8 correct responses from Trials $2-8$. With the stimulus below the response, the means for Experiments 1 and 2 were 9.6 and 10.9, respectively.

It must therefore be concluded from these data that employment of a standard left-right display leads to phenomena which are consistent with a transition from a stage of response consolidation to one of $S-R$ hook-up, but which are, at the same time, reflecting S's preference to read from left to right. The position of the stimulus in relation to that of the response is a significant feature of the data, as one would expect, but even with the material presented to afford maximum interference, the data are still supportive of the two-stage notion.

\section{REFERENCES}

McCORMACK, P. D., \& HALTRECHT, E. J. Monitoring eye movements under two conditions of presentations of paired-associate materials. Canadian Journal of Psychology, 1966, 20, 154-159.

MoCORMACK, P. D., HALTRECHT, E. J., \& HANNAH, T. E. Monitoring eye movements in nonlearning situations. Psychonomic Science, $1966,6,371-372$

NOBLE, C. E. Measurement of associative value (a), rated associations $\left(a^{\prime}\right)$ and scaled meaningfulness $\left(\mathrm{m}^{\prime}\right)$ for the $2100 \mathrm{CVC}$ combinations of the English alphabet. Psychological Reports, $1961,8,487-521$.

UNDERWOOD, B. J., \& SCHULZ, R. W. Meaningfulness and verbal learning. Chicago: Lippincott, 1960

\section{NOTE}

1. This research was supported by a grant-in-aid from the Associate Committee on Experimental Psychology of the National Research Council of Canada (Grant APA-78). 